\title{
ORTHOGONALITY OF DIVISORIAL ZARISKI DECOMPOSITIONS FOR CLASSES WITH VOLUME ZERO
}

\author{
VALENTINO TOSATTI
}

\begin{abstract}
We show that the orthogonality conjecture for divisorial Zariski decompositions on compact Kähler manifolds holds for pseudoeffective $(1,1)$ classes with volume zero.
\end{abstract}

\section{INTRODUCTION}

The orthogonality conjecture of divisorial Zariski decompositions [4] states the following:

Conjecture 1.1. Let $\left(X^{n}, \omega\right)$ be a compact Kähler manifold, and $\alpha$ a pseudoeffective $(1,1)$ class. Then

$$
\left\langle\alpha^{n-1}\right\rangle \cdot \alpha=\operatorname{Vol}(\alpha)
$$

Here $\operatorname{Vol}(\alpha)$ denotes the volume of the class $\alpha[1]$ and $\langle\cdot\rangle$ is the moving intersection product of classes as introduced by Boucksom [2, 4, 5, 6]. The name of this problem comes from the following observation. If we choose an approximate Zariski decomposition of the class $\alpha$ (see section 2 for the precise details of the construction), given by suitable modifications $\mu_{\delta}$ : $X_{\delta} \rightarrow X$, for all small $\delta>0$, with $\mu_{\delta}^{*}(\alpha+\delta \omega)=\theta_{\delta}+\left[E_{\delta}\right]$ with $\theta_{\delta}$ a semipositive class and $E_{\delta}$ an effective $\mathbb{R}$-divisor, then (1.1) is equivalent to

$$
\lim _{\delta \downarrow 0} \int_{X_{\delta}} \theta_{\delta}^{n-1} \wedge\left[E_{\delta}\right]=0,
$$

which explains the name. This conjecture was first raised by Nakamaye [14, p.566] when $X$ is projective and $\alpha=c_{1}(L)$ for some line bundle $L$. This was solved by Boucksom-Demailly-Păun-Peternell [4] who more generally proved Conjecture 1.1 when $X$ is projective and $\alpha$ belongs to the real Néron-Severi group, and posed it in the general case. It was observed in [4, 6] that Conjecture 1.1 is equivalent to several other powerful statements, including the fact that the dual cone of the pseudoeffective cone $\mathcal{E}$ is the movable cone $\mathcal{M}$, the weak transcendental Morse inequalities, and the $C^{1}$ differentiability of the volume function on the big cone. Very recently, Witt-Nyström [19] has proved Conjecture 1.1 when $X$ is projective.

Our main result is the following:

Supported in part by a Sloan Research Fellowship and NSF grant DMS-1308988. I am grateful to John Lesieutre for useful discussions and to Xiaokui Yang for helpful comments. 
Theorem 1.2. Conjecture 1.1 holds if $\operatorname{Vol}(\alpha)=0$.

The strategy of proof is similar to the one in [4] with one crucial difference. Since the weak transcendental Morse inequality

$$
\operatorname{Vol}(\alpha-\beta) \geqslant \int_{X} \alpha^{n}-n \int_{X} \alpha^{n-1} \wedge \beta,
$$

for the difference of two nef classes $\alpha, \beta$ remains conjectural, we employ instead the weaker version

$$
\operatorname{Vol}(\alpha-\beta) \geqslant \frac{\left(\int_{X} \alpha^{n}-n \int_{X} \alpha^{n-1} \wedge \beta\right)^{n}}{\left(\int_{X} \alpha^{n}\right)^{n-1}},
$$

which was proved independently in [18, 17, using the mass concentration technique of Demailly-Păun [10] and its recent improvements by Chiose [7, Xiao [20] and Popovici [16]. Inequality (1.3) is too weak to prove Conjecture 1.1 in general, but the fact that the numerator on the RHS has the correct form turns out to be enough to prove Theorem 1.2.

If we define the "difference function" $\mathcal{D}: \mathcal{E} \rightarrow \mathbb{R}$ by

$$
\mathcal{D}(\alpha):=\left\langle\alpha^{n-1}\right\rangle \cdot \alpha-\operatorname{Vol}(\alpha),
$$

then Conjecture 1.1 simply states that $\mathcal{D}$ vanishes identically on $\mathcal{E}$. As a corollary of Theorem 1.2 we have:

Corollary 1.3. The function $\mathcal{D}: \mathcal{E} \rightarrow \mathbb{R}$ is nonnegative, continuous on $\mathcal{E}$, and vanishes on its boundary.

Theorem 1.2 and Corollary 1.3 are proved in section 2, In section 3 we will make some further remarks on the function $\mathcal{D}$, and on the relation between Theorem 1.2 and the "cone duality" conjecture.

\section{THE MAIN THEOREM}

In this section we give the proof of Theorem 1.2 and Corollary 1.3 .

Proof of Theorem 1.2. Let $\alpha$ be any pseudoeffective $(1,1)$ class on $X$. By definition of moving intersection products, for any $1 \leqslant p \leqslant n$ the real $(p, p)$ cohomology class $\left\langle\alpha^{p}\right\rangle$ is defined to be

$$
\left\langle\alpha^{p}\right\rangle=\lim _{\delta \downarrow 0}\left\langle(\alpha+\delta \omega)^{p}\right\rangle,
$$

where for $\delta>0$ the class $\alpha+\delta \omega$ is big, and in this case we define

$$
\left\langle(\alpha+\delta \omega)^{p}\right\rangle=\left[\left\langle T_{\min , \delta}^{p}\right\rangle\right],
$$

where $T_{\min , \delta}$ is any positive current with minimal singularities in the class $\alpha+\delta \omega$, and $\left\langle T_{m i n, \delta}^{p}\right\rangle$ denotes the non-pluripolar product [5].

The volume of $\alpha$ (see [1]) is in fact equal to the moving self-intersection product

$$
\operatorname{Vol}(\alpha)=\left\langle\alpha^{n}\right\rangle=\lim _{\delta \downarrow 0}\left\langle(\alpha+\delta \omega)^{n}\right\rangle
$$


We now review the well-known construction of approximate Zariski decompositions [2, 4, 5, 6], following roughly the argument in [5, Proposition 1.18]. Applying Demailly's regularization [9] to $T_{\min , \frac{\delta}{2}}$ we obtain a sequence of currents $T_{\delta, \varepsilon}, \varepsilon>0$, in the big class $\alpha+\frac{\delta}{2} \omega$, with analytic singularities, with $T_{\delta, \varepsilon} \geqslant-\varepsilon \omega$, and with their potentials decreasing to that of $T_{\min , \frac{\delta}{2}}$ as $\varepsilon \rightarrow 0$. As long as $\varepsilon \leqslant \frac{\delta}{2}$, we have that $T_{\delta, \varepsilon}+\frac{\delta}{2} \omega$ and $T_{m i n, \frac{\delta}{2}}+\frac{\delta}{2} \omega$ are closed positive currents in the class $\alpha+\delta \omega$ whose potentials are locally bounded away from the proper analytic subvariety $A:=E_{n K}\left(\alpha+\frac{\delta}{2} \omega\right)$, and so by weak continuity of the Bedford-Taylor Monge-Ampère operator along decreasing sequences we have

$$
\left(T_{\delta, \varepsilon}+\frac{\delta}{2} \omega\right)^{p} \rightarrow\left(T_{\min , \frac{\delta}{2}}+\frac{\delta}{2} \omega\right)^{p}
$$

weakly on $X \backslash A$ as $\varepsilon \rightarrow 0$, for all $1 \leqslant p \leqslant n$. It follows that

$$
\begin{aligned}
\int_{X \backslash A}\left(T_{\min , \frac{\delta}{2}}+\frac{\delta}{2} \omega\right)^{p} \wedge \omega^{n-p} & \leqslant \liminf _{\varepsilon \rightarrow 0} \int_{X \backslash A}\left(T_{\delta, \varepsilon}+\frac{\delta}{2} \omega\right)^{p} \wedge \omega^{n-p} \\
& \leqslant \int_{X \backslash A}\left(T_{\min , \delta}\right)^{p} \wedge \omega^{n-p}
\end{aligned}
$$

where the last inequality follows from [5, Theorem 1.16], since all the currents involved have small unbounded locus. But as $\delta \rightarrow 0$ both the LHS and the RHS converge to

$$
\int_{X}\left\langle\alpha^{p}\right\rangle \wedge \omega^{n-p}
$$

and so we may choose a sequence $\varepsilon(\delta) \rightarrow 0$ such that the currents $T_{\delta}:=$ $T_{\delta, \varepsilon(\delta)}-\frac{\delta}{2} \omega$ in the class $\alpha$ have analytic singularities, satisfy $T_{\delta} \geqslant-\delta \omega$, and are such that

$$
\lim _{\delta \downarrow 0}\left\langle\left(T_{\delta}+\delta \omega\right)^{p}\right\rangle=\left\langle\alpha^{p}\right\rangle
$$

for all $1 \leqslant p \leqslant n$. Let $\mu_{\delta}: X_{\delta} \rightarrow X$ be a resolution of the singularities of $T_{\delta}+\delta \omega$, so that

$$
\mu_{\delta}^{*}\left(T_{\delta}+\delta \omega\right)=\theta_{\delta}+\left[E_{\delta}\right]
$$

so that $\theta_{\delta}$ is a smooth semipositive form, $E_{\delta}$ is an effective $\mathbb{R}$-divisor and $\left[E_{\delta}\right]$ denotes the current of integration. We refer to this construction as an approximate Zariski decomposition for the class $\alpha$.

As discussed above, we have

$$
\operatorname{Vol}(\alpha)=\lim _{\delta \downarrow 0} \int_{X}\left\langle\left(T_{\delta}+\delta \omega\right)^{n}\right\rangle=\lim _{\delta \downarrow 0} \int_{X_{\delta}}\left\langle\left(\mu_{\delta}^{*}\left(T_{\delta}+\delta \omega\right)\right)^{n}\right\rangle=\lim _{\delta \downarrow 0} \int_{X_{\delta}} \theta_{\delta}^{n},
$$




$$
\begin{aligned}
\left\langle\alpha^{n-1}\right\rangle \cdot \alpha & =\lim _{\delta \downarrow 0} \int_{X}\left\langle\left(T_{\delta}+\delta \omega\right)^{n-1}\right\rangle \wedge \alpha=\lim _{\delta \downarrow 0} \int_{X}\left\langle\left(T_{\delta}+\delta \omega\right)^{n-1}\right\rangle \wedge(\alpha+\delta \omega) \\
& =\lim _{\delta \downarrow 0} \int_{X_{\delta}}\left\langle\left(\mu_{\delta}^{*}\left(T_{\delta}+\delta \omega\right)\right)^{n-1}\right\rangle \wedge \mu_{\delta}^{*}(\alpha+\delta \omega) \\
& =\lim _{\delta \downarrow 0} \int_{X_{\delta}}\left(\theta_{\delta}^{n}+\theta_{\delta}^{n-1} \wedge\left[E_{\delta}\right]\right),
\end{aligned}
$$

so that the orthogonality relation (1.1) is in general equivalent to the statement that

$$
\lim _{\delta \downarrow 0} \int_{X_{\delta}} \theta_{\delta}^{n-1} \wedge\left[E_{\delta}\right]=0 .
$$

We now follow [4], and fix a constant $C_{0}$ such that $C_{0} \omega \pm(\alpha+\delta \omega)$ is nef. We write

$$
E_{\delta}=\mu_{\delta}^{*}\left(\alpha+\delta \omega+C_{0} \omega\right)-\left(\theta_{\delta}+C_{0} \mu_{\delta}^{*} \omega\right),
$$

as the difference of two nef classes. For $t \in[0,1]$ write

$$
\theta_{\delta}+t E_{\delta}=A-B
$$

where

$$
\begin{gathered}
A=\theta_{\delta}+t \mu_{\delta}^{*}\left(\alpha+\delta \omega+C_{0} \omega\right), \\
B=t\left(\theta_{\delta}+C_{0} \mu_{\delta}^{*} \omega\right),
\end{gathered}
$$

and $A, B$ are nef. Then

$$
\operatorname{Vol}(\alpha+\delta \omega)=\operatorname{Vol}\left(\theta_{\delta}+E_{\delta}\right) \geqslant \operatorname{Vol}\left(\theta_{\delta}+t E_{\delta}\right)=\operatorname{Vol}(A-B) .
$$

We use [18, Theorem 1.1] (also independently obtained in [17, Theorem 3.5]) and obtain

$$
\operatorname{Vol}(A-B) \geqslant \frac{\left(\int_{X_{\delta}} A^{n}-n \int_{X_{\delta}} A^{n-1} \wedge B\right)^{n}}{\left(\int_{X_{\delta}} A^{n}\right)^{n-1}} .
$$

We follow the same argument as in [4, p.218] (see also [12, (11.15)]) and estimate

$$
\int_{X_{\delta}} A^{n}-n \int_{X_{\delta}} A^{n-1} \wedge B \geqslant \int_{X_{\delta}} \theta_{\delta}^{n}+n t \int_{X_{\delta}} \theta_{\delta}^{n-1} \wedge\left[E_{\delta}\right]-5 n^{2} t^{2} C_{0}^{n} \int_{X} \omega^{n},
$$

as long as $t \leqslant \frac{1}{10 n}$. We choose

$$
t=\frac{\int_{X_{\delta}} \theta_{\delta}^{n-1} \wedge\left[E_{\delta}\right]}{10 n C_{0}^{n} \int_{X} \omega^{n}}
$$

which is easily seen to be less than $\frac{1}{10 n}$, and so we obtain

$$
\int_{X_{\delta}} A^{n}-n \int_{X_{\delta}} A^{n-1} \wedge B \geqslant \int_{X_{\delta}} \theta_{\delta}^{n}+\frac{1}{20} \frac{\left(\int_{X_{\delta}} \theta_{\delta}^{n-1} \wedge\left[E_{\delta}\right]\right)^{2}}{C_{0}^{n} \int_{X} \omega^{n}},
$$


and plugging this into (2.2) we obtain

$$
\operatorname{Vol}(\alpha+\delta \omega) \geqslant \frac{\left(\int_{X_{\delta}} \theta_{\delta}^{n}+\frac{1}{20} \frac{\left(\int_{X_{\delta}} \theta_{\delta}^{n-1} \wedge\left[E_{\delta}\right]\right)^{2}}{C_{0}^{n} \int_{X} \omega^{n}}\right)^{n}}{\left(\int_{X_{\delta}} A^{n}\right)^{n-1}} .
$$

We also have

$$
\int_{X_{\delta}} A^{n}=\int_{X_{\delta}}\left(\theta_{\delta}+t \mu_{\delta}^{*}\left(\alpha+\delta \omega+C_{0} \omega\right)\right)^{n} \leqslant \int_{X_{\delta}} \theta_{\delta}^{n}+C t \leqslant C,
$$

and so

$$
\int_{X_{\delta}} \theta_{\delta}^{n}+\frac{1}{20} \frac{\left(\int_{X_{\delta}} \theta_{\delta}^{n-1} \wedge\left[E_{\delta}\right]\right)^{2}}{C_{0}^{n} \int_{X} \omega^{n}} \leqslant C \operatorname{Vol}(\alpha+\delta \omega)^{\frac{1}{n}} \rightarrow C \operatorname{Vol}(\alpha)^{\frac{1}{n}}=0,
$$

as $\delta \rightarrow 0$, and we conclude that

$$
\int_{X_{\delta}} \theta_{\delta}^{n-1} \wedge\left[E_{\delta}\right] \rightarrow 0
$$

which proves (2.1).

Remark 2.1. Some of the estimates in this proof, such as for example (2.3), are far from being sharp. It is easy to make them sharp, but this does not appear to give any useful improvement.

Proof of Corollary 1.3. Using the notation as in the proof of Theorem 1.2, we have that

$$
\mathcal{D}(\alpha)=\lim _{\delta \downarrow 0} \int_{X_{\delta}} \theta_{\delta}^{n-1} \wedge\left[E_{\delta}\right] \geqslant 0 .
$$

It follows easily from the definitions that moving intersection products are upper-semicontinuous on the pseudoeffective cone and continuous in its interior (the big cone), while it was proved in [1] that the volume function is continuous on the whole pseudoeffective cone. It follows that $\mathcal{D}$ is continuous on the big cone and upper-semicontinuous on the pseudoeffective cone. By Theorem 1.2, $\mathcal{D}$ vanishes on its boundary, and hence it is continuous on all of $\mathcal{E}$.

\section{Further REMARKS}

In this section we collect some further remarks on the function $\mathcal{D}$ and on the cone duality conjecture of [4].

The function $\mathcal{D}$ defined in (1.4) clearly vanishes on the nef cone, where moving intersection products equal usual intersection products (see e.g. 5). More generally we have the following result, which is a simple consequence of the author's work with Collins [8] and was also observed by Deng [11]:

Proposition 3.1. Let $\alpha$ be a pseudoeffective $(1,1)$ class, and write $\alpha=$ $P+N$ for its divisorial Zariski decomposition. If the class $P$ is nef then $\mathcal{D}(\alpha)=0$. 
Recall here that the positive part is given by $P=\langle\alpha\rangle$, which is in general only nef in codimension 1 [3, 15].

Proof. First, we show that

$$
\left\langle\alpha^{n-1}\right\rangle \cdot N=0 .
$$

Since moving products are unchanged if we replace a class by its positive part (see [4, 6]), this is equivalent to showing that

$$
\left\langle P^{n-1}\right\rangle \cdot N=0,
$$

and since by assumption $P$ is nef, this is also equivalent to showing that

$$
P^{n-1} \cdot N=0 \text {. }
$$

But by definition the irreducible components of $N$ are contained in $E_{n K}(\alpha)=$ $E_{n K}(P)$ (see e.g. [13, Claim 4.7]), and so are also irreducible components of $E_{n K}(P)$. By the main theorem of [8] we have therefore $P^{n-1} \cdot N=0$. On the other hand since $P$ is nef we also have

$$
\left\langle\alpha^{n-1}\right\rangle \cdot \alpha=\left\langle\alpha^{n-1}\right\rangle \cdot P=\left\langle P^{n-1}\right\rangle \cdot P=P^{n}=\operatorname{Vol}(P)=\operatorname{Vol}(\alpha),
$$

as claimed.

Remark 3.2. In fact, it is not hard to see (cf. [4]) that in general (1.1) is equivalent to the following two relations both holding

$$
\begin{gathered}
\left\langle\alpha^{n-1}\right\rangle \cdot P=\operatorname{Vol}(\alpha), \\
\left\langle\alpha^{n-1}\right\rangle \cdot N=0 .
\end{gathered}
$$

It was proved in [4 that Conjecture 1.1] implies (and is in fact equivalent to) the "cone duality" conjecture, which states that the dual cone of the pseudoeffective cone $\mathcal{E}$ of a compact Kähler manifold equals the movable cone $\mathcal{M} \subset H^{n-1, n-1}(X, \mathbb{R})$. It is easy to see that $\mathcal{E} \subset \mathcal{M}^{\vee}$, so the point is to prove the reverse inclusion. The proof given there starts by assuming that there is a class $\alpha \in \partial \mathcal{E} \cap\left(\mathcal{M}^{\vee}\right)^{\circ}$, and derives a contradiction, assuming that $X$ is projective and that the class belongs to the real Néron-Severi group.

In general we have the following:

Proposition 3.3. Let $\alpha \in \partial \mathcal{E} \cap\left(\mathcal{M}^{\vee}\right)^{\circ}$. Then we must have $\left\langle\alpha^{n-1}\right\rangle=0$.

Proof. Assume for a contradiction that $\left\langle\alpha^{n-1}\right\rangle \neq 0$ in $H^{n-1, n-1}(X, \mathbb{R})$. Since this class is represented by a closed nonnegative $(n-1, n-1)$ current, it follows that

$$
\int_{X}\left\langle\alpha^{n-1}\right\rangle \wedge \omega>0
$$

where $\omega$ is a fixed Kähler metric. We choose an approximate Zariski decomposition $T_{\delta}$ with resolutions $\mu_{\delta}: X_{\delta} \rightarrow X$ as before, so that we have also

$$
0<2 \eta \leqslant \int_{X}\left\langle\alpha^{n-1}\right\rangle \wedge \omega=\lim _{\delta \downarrow 0} \int_{X_{\delta}} \theta_{\delta}^{n-1} \wedge \mu_{\delta}^{*} \omega=\lim _{\delta \downarrow 0} \int_{X}\left(\mu_{\delta}\right)_{*}\left(\theta_{\delta}^{n-1}\right) \wedge \omega,
$$


for some fixed $\eta>0$. Up to modifying the classes $\theta_{\delta}$ (and $\left[E_{\delta}\right]$ ) by subtracting a small multiple of $\operatorname{Exc}\left(\mu_{\delta}\right)$, we may also assume that the classes $\theta_{\delta}$ are Kähler on $X_{\delta}$, so that the pushforwards $\left(\mu_{\delta}\right)_{*}\left(\theta_{\delta}^{n-1}\right)$ are movable classes on $X$.

Let $\varepsilon>0$ be such that $\alpha-\varepsilon \omega \in \mathcal{M}^{\vee}$. Integrating $\alpha-\varepsilon \omega$ against the class $\left(\mu_{\delta}\right)_{*}\left(\theta_{\delta}^{n-1}\right) \in \mathcal{M}$ we obtain

$$
\int_{X} \alpha \wedge\left(\mu_{\delta}\right)_{*}\left(\theta_{\delta}^{n-1}\right) \geqslant \varepsilon \int_{X} \omega \wedge\left(\mu_{\delta}\right)_{*}\left(\theta_{\delta}^{n-1}\right) \geqslant \varepsilon \eta>0,
$$

for all $\delta>0$ small. But we also have

$$
\int_{X} \alpha \wedge\left(\mu_{\delta}\right)_{*}\left(\theta_{\delta}^{n-1}\right)=\int_{X_{\delta}} \mu_{\delta}^{*} \alpha \wedge \theta_{\delta}^{n-1} \leqslant \int_{X_{\delta}} \mu_{\delta}^{*}(\alpha+\delta \omega) \wedge \theta_{\delta}^{n-1}=\int_{X_{\delta}}\left(\theta_{\delta}^{n}+\theta_{\delta}^{n-1} \wedge\left[E_{\delta}\right]\right),
$$

and putting these together we have

$$
\int_{X_{\delta}}\left(\theta_{\delta}^{n}+\theta_{\delta}^{n-1} \wedge\left[E_{\delta}\right]\right) \geqslant \varepsilon \eta
$$

for all $\delta>0$ small. Since $\operatorname{Vol}(\alpha)=0$, this implies

$$
\int_{X_{\delta}} \theta_{\delta}^{n-1} \wedge\left[E_{\delta}\right] \geqslant \frac{\varepsilon \eta}{2}
$$

for all $\delta>0$ small, which is a contradiction to Theorem 1.2, Therefore we must have that $\left\langle\alpha^{n-1}\right\rangle=0$.

Remark 3.4. Even though the class $\alpha \in \partial \mathcal{E} \cap\left(\mathcal{M}^{\vee}\right)^{\circ}$ does satisfy the orthogonality conjecture (say as in (2.1) ) by Theorem 1.2, because it has volume zero, this is not enough to derive a contradiction in general. Indeed, to make the argument in [4] go through, one would need the following quantitative version of orthogonality

$$
\left(\int_{X_{\delta}} \theta_{\delta}^{n-1} \wedge\left[E_{\delta}\right]\right)^{2} \leqslant C\left(\operatorname{Vol}(\alpha+\delta \omega)-\int_{X_{\delta}} \theta_{\delta}^{n}\right),
$$

(cf. [4, Theorem 4.1]) which does not follow from the arguments of Theorem 1.2 .

\section{REFERENCES}

[1] S. Boucksom On the volume of a line bundle, Internat. J. Math. 13 (2002), no. 10, 1043-1063.

[2] S. Boucksom Cônes positifs des variétés complexes compactes, Ph.D. Thesis, Institut Fourier Grenoble, 2002.

[3] S. Boucksom Divisorial Zariski decompositions on compact complex manifolds, Ann. Sci. École Norm. Sup. (4) 37 (2004), no. 1, 45-76.

[4] S. Boucksom, J.-P. Demailly, M. Păun, T. Peternell The pseudo-effective cone of a compact Kähler manifold and varieties of negative Kodaira dimension, J. Algebraic Geom. 22 (2013), no. 2, 201-248.

[5] S. Boucksom, P. Eyssidieux, V. Guedj, A. Zeriahi Monge-Ampère equations in big cohomology classes, Acta Math. 205 (2010), no. 2, 199-262.

[6] S. Boucksom, C. Favre, M. Jonsson Differentiability of volumes of divisors and a problem of Teissier, J. Algebraic Geom. 18 (2009), no. 2, 279-308. 
[7] I. Chiose The Kähler rank of compact complex manifolds, J. Geom. Anal. 26 (2016), no. $1,603-615$.

[8] T.C. Collins, V. Tosatti Kähler currents and null loci, Invent. Math. 202 (2015), no.3, 1167-1198.

[9] J.-P. Demailly Regularization of closed positive currents and intersection theory, J. Algebraic Geom. 1 (1992), no. 3, 361-409.

[10] J.-P. Demailly, M. Păun Numerical characterization of the Kähler cone of a compact Kähler manifold, Ann. of Math., 159 (2004), no. 3, 1247-1274.

[11] Y. Deng Transcendental Morse inequality and generalized Okounkov bodies, arXiv:1503.00112

[12] R. Lazarsfeld Positivity in algebraic geometry I $\&$ II, Springer-Verlag, Berlin, 2004.

[13] S. Matsumura Restricted volumes and divisorial Zariski decompositions, Amer. J. Math. 135 (2013), no. 3, 637-662.

[14] M. Nakamaye Base loci of linear series are numerically determined, Trans. Amer. Math. Soc. 355 (2003), no. 2, 551-566.

[15] N. Nakayama Zariski-decomposition and abundance, MSJ Memoirs, 14. Mathematical Society of Japan, Tokyo, 2004.

[16] D. Popovici Sufficient bigness criterion for differences of two nef classes, Math. Ann. 364 (2016), no. 1-2, 649-655.

[17] D. Popovici Volume and self-intersection of differences of two nef classes, arXiv:1505.03457.

[18] V. Tosatti The Calabi-Yau Theorem and Kähler currents, arXiv:1505.02124.

[19] D. Witt-Nyström Duality between the pseudoeffective and the movable cone on a projective manifold. With an appendix by Sébastien Boucksom, arXiv:1602.03778.

[20] J. Xiao Weak transcendental holomorphic Morse inequalities on compact Kähler manifolds, Ann. Inst. Fourier (Grenoble) 65 (2015), no. 3, 1367-1379.

Department of Mathematics, Northwestern University, 2033 Sheridan Road, EvANSTON, IL 60208

E-mail address: tosatti@math.northwestern.edu 